M. Schewe*, D. Kohlmann, H. Wulfmeier, H. Fritze und C. Rembe

\title{
Methoden zur Minimierung des Rauscheinflusses durch Hitzeflimmern bei einem heterodynen Laser-Doppler-Vibrometer
}

\author{
Methods to minimize the noise contribution through heat haze with a heterodyne laser-Doppler \\ vibrometer
}

DOI 10.1515/teme-2020-0023

Zusammenfassung: Bei der kontaktlosen Schwingungsmessung im Hochtemperaturbereich mit einem heterodynen Laser-Doppler-Vibrometer begrenzen insbesondere im Frequenzbreich unter $50 \mathrm{~Hz}$ mehrere Faktoren die minimal auflösbaren Schwingungsamplituden. In diesem Beitrag werden der Einfluss des Hitzeflimmerns auf den Rauschpegel analysiert und Methoden vorgestellt, wie dieser Einfluss minimiert werden kann. Darüber hinaus werden weitere begrenzende Faktoren wie der Einfluss der Laufzeitunterschiede der Laserstrahlen sowie Lösungen zu deren Minimierung vorgestellt.

Schlüsselwörter: heterodyne Laser-Doppler-Vibrometrie, differentielles Messen, Hochtemperatur-Aktoren, niederfrequente Schwingungen, optische Turbulenz

\begin{abstract}
For contactless vibration measurement with a laser-Doppler vibrometer in the high-temperature range, especially in the frequency range below $50 \mathrm{~Hz}$, multiple factors limit the minimally detectable displacement amplitude. In this article the impact of heat haze on the noise level is analyzed and methods are presented on how this impact can be minimized. Additional limiting factors, like the effect of the time-of-flight difference between the laser beams, and possible solutions to reduce the effect of those factors are presented.
\end{abstract}

Keywords: heterodyne laser-Doppler vibrometry, differential measurement, high temperature actors, low frequency vibrations, optical turbulence

\footnotetext{
*Korrespondenzautor: M. Schewe, Institut für Elektrische Informationstechnik, TU Clausthal, Leibnizstraße 28, 38678 Clausthal-Zellerfeld, schewe@iei.tu-clausthal.de D. Kohlmann, H. Wulfmeier, H. Fritze, Institut für Energieforschung und Physikalische Technologien, TU Clausthal, Am Stollen 19b, 38640 Goslar

C. Rembe, Institut für Elektrische Informationstechnik, TU Clausthal, Leibnizstraße 28, 38678 Clausthal-Zellerfeld
}

\section{Einleitung}

In den letzten Jahren haben sich Laser-DopplerVibrometer (LDV) für die Schwingungsmessung in der Industrie etabliert. Durch den geringen Montageaufwand sowie die Möglichkeit, kontaktlos auf sich drehenden oder heißen Oberflächen zu messen, werden immer weitere Anwendungsfelder erschlossen. Die hohe Sensitivität erlaubt zudem die Messung von Schwingungsamplituden im Pikometer-Bereich [2] in einer breiten Frequenzspanne. Im unteren Frequenzbereich, d. h. unterhalb $50 \mathrm{~Hz}$, ergibt sich hierbei ein Rauschniveau von bis zu $100 \mathrm{~nm} / \sqrt{\mathrm{Hz}}$, das durch die von Umwelteinflüssen wie Wärme, Wind oder Erschütterungen hervorgerufenen optischen Weglängenunterschiede erzeugt wird [3]. Aufgrund der geringen Relevanz für die üblichen technischen Anwendungen wurden die Empfangselektronik und die Demodulatoren für diesen Frequenzbereich noch nicht auf die Schrotrauschbegrenzung optimiert.

Ansätze zur Modellierung der Einflüsse von Turbulenzen in der Luft auf die Ausbreitung eines Laserstrahls existieren hingegen bereits. Bei einer Temperaturdifferenz von $150 \mathrm{~K}$ über einer Distanz von $10 \mathrm{~cm}$ entstehen beispielsweise Störungen im Frequenzbereich von 3-6 Hz. [7]

Ein aktuelles Forschungsgebiet ist die Untersuchung der chemischen Expansion von Praseodym-CerMischoxidschichten (PCO) bei niedrigen Frequenzen. Diese Schichten haben das Potential als HochtemperaturAktoren eingesetzt zu werden. Bisher wurde die chemische Expansion dieser Schichten im Bereich von $0,2 \mathrm{~Hz}$ bis $5 \mathrm{~Hz}$ analysiert. Dabei wurde bei $1 \mathrm{~Hz}$ eine maximale Ausdehnung von über $18 \mathrm{~nm}$ in der Mitte einer Probe detektiert [6]. Allerdings ist eine Verbesserung der Aufösung im Bereich unter $1 \mathrm{~nm}$ für Frequenzen unter $1 \mathrm{~Hz}$ für unsere Anwendung erforderlich, um die Reaktionskinetik in den Aktorschichten charakterisieren zu können. Niederfrequente Störungen werden in dieser Anwendung bei 
hohen Temperaturen durch Brechungsindexschwankungen der Luft erzeugt [4] und begrenzen hierbei die minimal auflösbaren Schwingungsamplituden unterhalb von $10 \mathrm{~Hz}$ Eine Störung, die diese Schwankungen verursacht, ist als Hitzeflimmern bekannt.

\section{Methode}

\subsection{Quantifizierung des Rauscheinflusses durch Hitzeflimmern}

Um die Störeinflüsse der Umgebung - primär die des Hitzeflimmerns - zu quantifizieren, wird eine Probe $14 \mathrm{~cm}$ tief, mittig in einem Rohrofen befestigt und schrittweise aufgeheizt. Zur Messung der Temperatur der Probe, wird ein Temperatursensor in unmittelbarer Nähe befestigt. Der Aufbau für die Messungen ist in Abb. 1 dargestellt. Der Laserspot eines selbstentwickelten LDV mit uns bekannten Rauscheigenschaften wird mit Hilfe von zwei Spiegeln auf der Probe in ca. $70 \mathrm{~cm}$ Entfernung ausgerichtet. Der Aufbau entspricht dabei dem eines standardmäßigen heterodynen LDV, wie z. B. in [5] dargestellt. Der Abstand zwischen dem LDV und dem Rohrofen beträgt in diesem Fall ca. $45 \mathrm{~cm}$. Nachdem die jeweils eingestell-

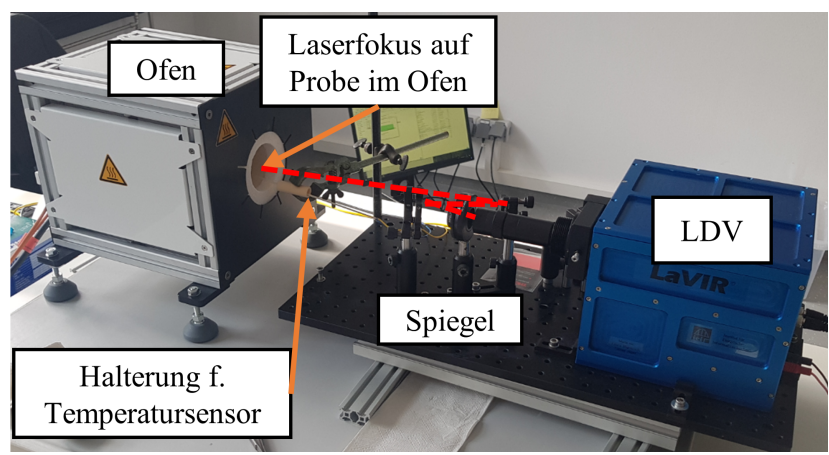

Abb. 1: Aufbau zur Messung des Temperatureinflusses auf den Rauschpegel eines selbstgebauten LDV.

te Temperatur konstant bleibt, werden Messungen bei Raumtemperatur sowie Probentemperaturen von $147^{\circ} \mathrm{C}$, $316^{\circ} \mathrm{C}, 490^{\circ} \mathrm{C}$ und $700^{\circ} \mathrm{C}$, durchgeführt. Um eine möglichst starke Konvektion der Luft und Hitzeflimmern im Bereich der Rohröffnung zu erzeugen, wird der Rohrofen einseitig offen gelassen. Die Auswertung der Messungen zeigt bei Erhöhung der Temperatur einen deutlichen Anstieg des Rauschpegels im Frequenzbereich unter $50 \mathrm{~Hz}$. Oberhalb dieser Frequenz beeinflussen andere, vermutlich temperaturinvariante Störfaktoren wie z. B. Vibrationen durch Gerätelüfter, Bewegungen durch Personen, etc., den Rauschpegel stärker. Die Ergebnisse der Messungen sind in Abb. 2 dargestellt und ermöglichen eine erste Einschätzung des Einflusses der resultierenden Störung auf die minimal auflösbare Schwingungsamplitude. Im Bereich unter $50 \mathrm{~Hz}$ sind Amplituden bei Raumtemperatur bereits ab ca. 500 pm auflösbar, wohingegen bei einer Probentemperatur von $700{ }^{\circ} \mathrm{C}$ Amplituden erst ab ca. $3 \mathrm{~nm}$ auflösbar sind.

\subsection{Einfluss differentiellen Messens auf den Rauschpegel des Hitzeflimmerns}

Um Störungen durch thermische und weitere Umwelteinflüsse zu reduzieren, wurde von uns ein differentiell messendes LDV gebaut. Um den Effekt zu untersuchen, werden sowohl Mess- als auch Referenzstrahl durch die heiße Luft geführt. Da beide weitgehend dieselben Störungen erfahren, wird davon ausgegangen, dass deren Einfluss durch Differenzbildung im Messsignal deutlich reduziert werden kann. Erste Tests belegen, dass der Einfluss durch störende Vibrationen erheblich verringert wird. Im folgenden Abschnitt wird auch die Minimierung der Einflüsse der Brechungsindexfluktuationen durch das Hitzeflimmern nachgewiesen. Dazu erfolgt ein direkter Vergleich einer konventionellen Messung, bei dem der Referenzstrahl unbeeinflusst durch Störungen ist, und einer differentiellen Messung. Im Weiteren wird LDV als Bezeichnung für ein konventionelles Gerät und DLDV für das differentiell messende Gerät verwendet.

Für einen reproduzierbaren Vergleich einer LDV- und einer DLDV-Messung wird jeweils das differentiell messende Gerät verwendet, um identische Komponenten zu gewährleisten. Für das Experiment, dessen Aufbau in Abb. 3 gezeigt ist, wird eine Heizplatte auf $240^{\circ} \mathrm{C}$ erhitzt und unterhalb des Strahlenganges positioniert. Durch die Temperaturdifferenz entsteht dabei ein Hitzeflimmern, das zu einer Änderung des Brechungsindex der Luft führt und vom Vibrometer als störende Schwingung erfasst wird. Bei der LDV-Messung wird dabei nur der Messstrahl durch die erhitzte Luft auf einer Reflektorfolie positioniert, während der Referenzstrahl auf einen räumlich getrennten Bereich mit Reflektorfolie geführt wird. Dies ist nur für unseren Aufbau notwendig, da sich bei einem konventionellen Gerät der Strahl direkt im Gerät befindet. Bei der DLDV-Messung werden beide Strahlen durch die erhitzte Luft auf derselben Reflektorfolie positioniert.

Beim Aufbau muss darauf geachtet werden, dass der zurückgelegte Weg des Referenzstrahls bei der LDV- bzw. DLDV-Messung vergleichbar ist. Der Weglängenunter- 


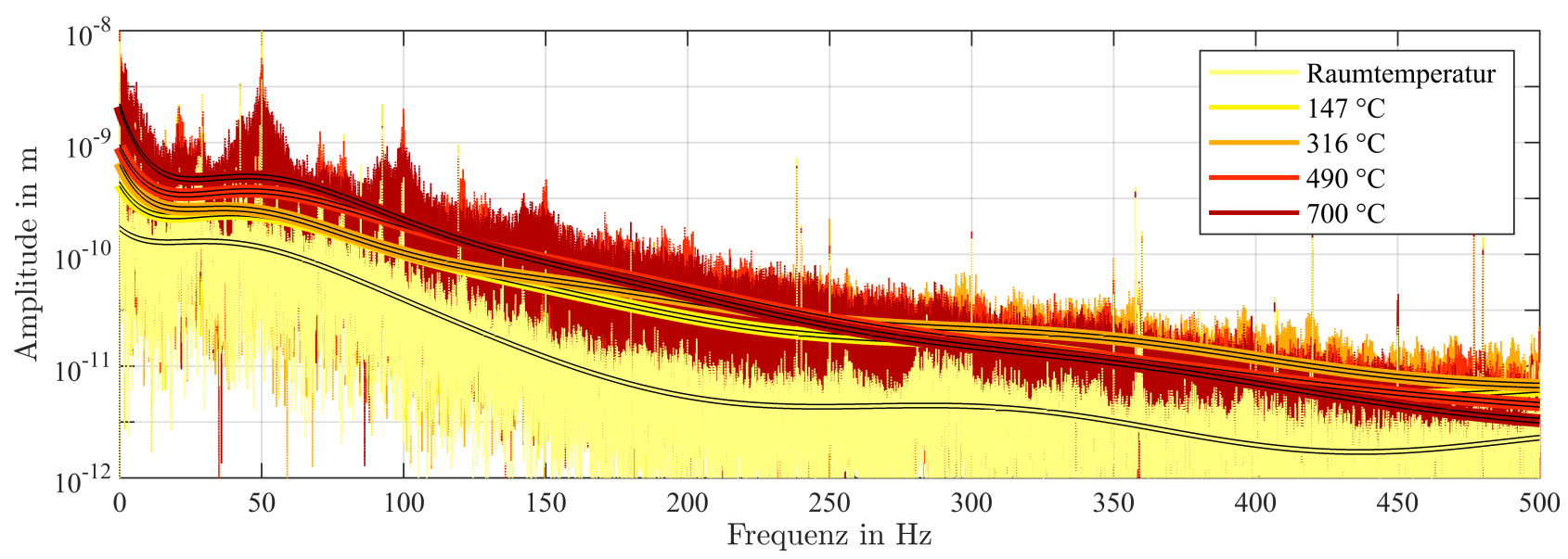

Abb. 2: Rauschpegel eines LDV im Frequenzbereich bis $500 \mathrm{~Hz}$ bei unterschiedlichen Probentemperaturen. Zur Visualisierung wurde jeweils eine Trendlinie (polynomische Regression 20. Grades, mit schwarzer Umrandung) eingefügt.

schied des Referenz- und des Messstrahls beträgt bei diesem Aufbau einige Zentimeter. Die Relevanz dessen wird im Kapitel 3.1.1 erklärt. Mit dem in Abb. 3 gezeigten Aufbau wird jeweils eine Messung ohne die Heizplatte aufgenommen und mehrere Messungen mit der Heizplatte. Dabei wird die Position des bzw. der Laserstrahlen auf der Folie variiert. Bei den LDV-Messungen verändert

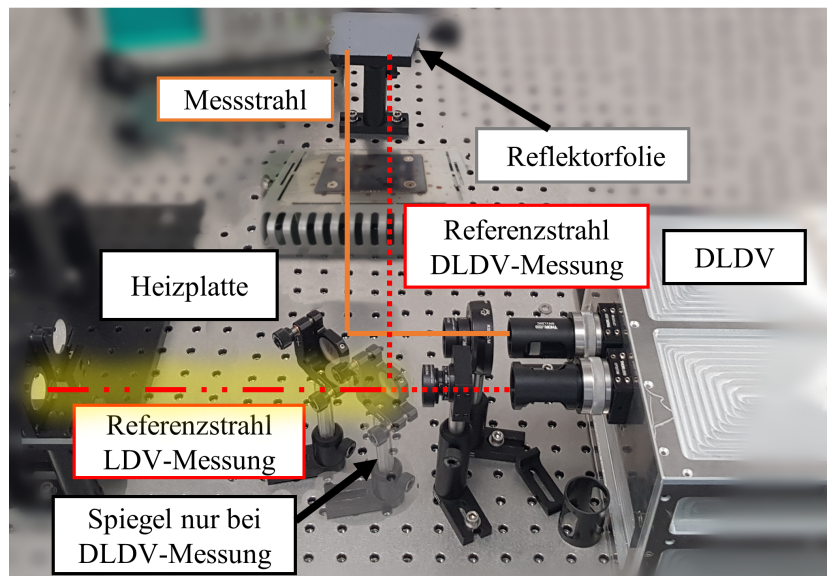

Abb. 3: Testaufbau zum Vergleich einer LDV- und einer DLDVMessung in Bezug auf den Einfluss der Lufterwärmung durch eine Heizplatte.

sich der Rauschpegel dabei unmerklich, wohingegen er bei den DLDV-Messungen, je nach Abstand $\Delta a$ der beiden Strahlen voneinander, stark beeinflusst werden kann. Die Ergebnisse dieser Messungen sind in Abb. 4 gezeigt.

\section{Ergebnisse}

Aus den in Abb. 4 gezeigten Messungen ist erkennbar, dass der Rauschpegel einer DLDV-Messung (in Dunkelgrau) geringer ist als der einer LDV-Messung (in Rot). Bei beiden Messmethoden steigt der Pegel bei Erhitzung der Luft durch die die Heizplatte deutlich an. Bei dem so entstehenden Hitzeflimmern liegt der Pegel der DLDVMessungen bei einem geringen Abstand $\Delta a<12 \mathrm{~mm}$ (in Grün) zwischen Mess- und Referenzstrahl deutlich unter dem der LDV-Messung (in rot gestrichelt). Die anfänglich getroffene Vermutung konnte hiermit bestätigt und die Funktionalität des differentiellen Messens nachgewiesen werden.

Eine wichtige Erkenntnis ist, dass sich der Rauschpegel einer DLDV-Messung bei einem Abstand $\Delta a>11 \mathrm{~mm}$ verzehnfacht und bei $\Delta a>15 \mathrm{~mm}$ sogar über dem einer LDV-Messung liegt (in blau). Um dies erklären zu können, müssen die Ursachen der auftretenden Störung genauer betrachtet werden. Hierfür soll auf eine vereinfachte Betrachtung Kolmogorovs Theorie zu turbulenten Strömungen, wie z. B. in [1] ausgeführt, eingegangen werden. In dieser wird u. a. die Änderung des Brechungsindex der Luft durch Temperaturgradienten in der Erdatmosphäre beschrieben. Die dabei entstehenden Bereiche optischer Turbulenzen werden in einzelne Zellen aufgeteilt. Jede dieser Zellen kann vereinfacht als homogen angenommen werden. Das bedeutet, dass wir von einem nahezu konstanten Brechungsindex innerhalb einer Zelle ausgehen können. Die Größe dieser Zellen kann von einigen Millimetern bis hin zu mehreren Zentimetern variieren. Zusätzlich können sich diese Zellen auch überlagern und ihre Größe ändern [1]. Da in Kolmogorovs Theorie die Größe der Zellen für 


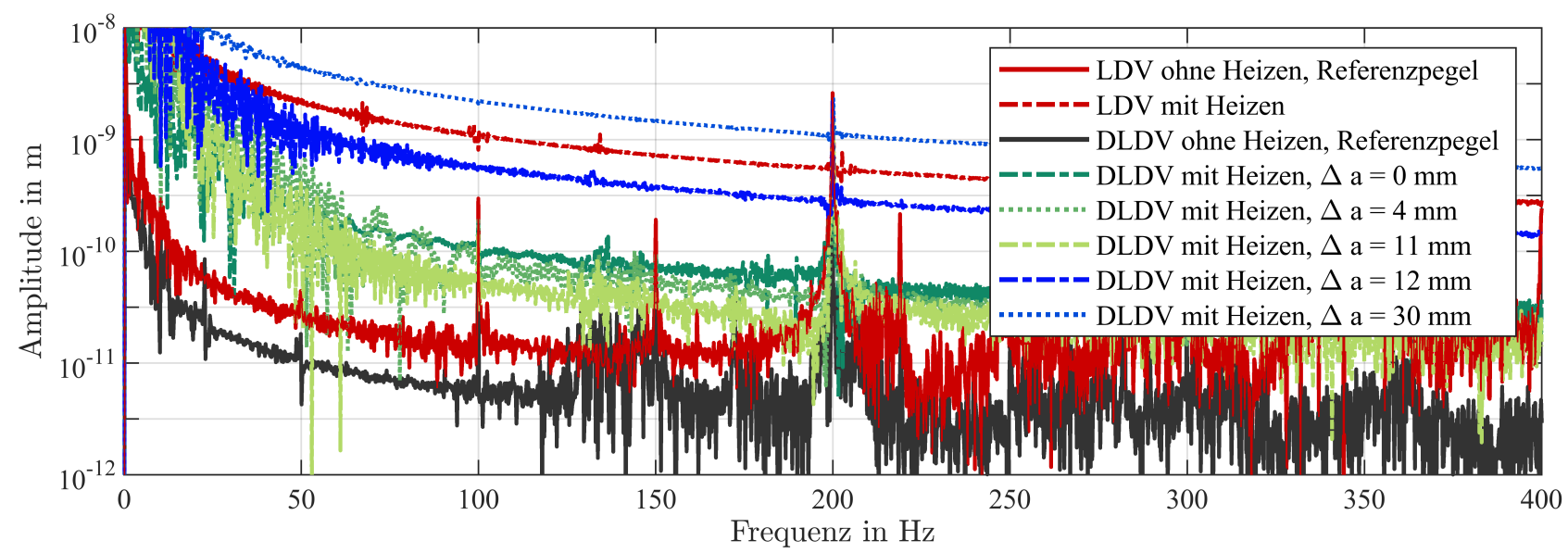

Abb. 4: Vergleich der Rauschpegel einer LDV- und einer DLDV-Messung bei Erhitzung der Luft durch eine Heizplatte bei Variation des Abstandes $\Delta a$ des Mess- und Referenzstrahls voneinander.

Effekte in der Atmosphäre beschrieben wird, lässt sie sich nur bedingt auf unsere Anwendung übertragen. Für eine prinzipielle Einordnung ist sie allerdings hinreichend. Auf die in Abb. 4 dargestellte Messung bezogen, kann davon ausgegangen werden, dass durch das Hitzeflimmern Zellen unterschiedlicher Größe entstehen. In diesen treten Brechungsindexfluktuationen auf, die von den hindurch geführten Laserstrahlen als Störung erfasst werden. Je kleiner der Abstand $\Delta a$ der beiden Strahlen hierbei ist, desto größer ist die Wahrscheinlichkeit, dass sie die gleiche Zelle durchlaufen und so die gleiche Störung erfahren. Anhand der Messergebnisse kann deshalb bei diesem Aufbau im Strahlengang eine Zellgröße von ca. $11 \mathrm{~mm}$ angenommen werden. In Realität ist die Entstehung von Zellen beliebig komplex. Dabei können beide Strahlen auch auf beiden Wegen durch stochastisch unabhängige Zellen geführt werden. Dabei wäre der resultierende Rauschpegel am höchsten, da sämtliche Störungen im resultierenden Signal additiv erfasst werden können. Somit lässt sich der höhere Rauschpegel bei Messungen mit einem größeren Abstand $\Delta a$ erklären.

Zur genaueren Betrachtung wird zukünftig für unsere Anwendung ein Modell zur Entstehung und zur Größe dieser Zellen erstellt oder experimentell ermittelt.

\subsection{Diskussion der Optimierung durch Laufzeitanpassung}

Im Folgenden werden weitere Störquellen unseres Aufbaus und Lösungen vorgestellt, um den Einfluss dieser Störungen zu minimieren.

Durch den experimentellen Aufbau unterscheiden sich die Weglängen des Mess- und des Referenzstrahls vonein- ander. Diese Differenz führt zu einem Zeitversatz der Laserstrahlen, durch den die Information über die Frequenzänderung durch die Doppler-Verschiebung, bzw. auch von Störungen, unterschiedlich schnell übertragen wird. Wird diese Weglängendifferenz nicht kompensiert, können auftretende Störungen nicht eliminiert werden. Bei unserem Aufbau kann die Notwendigkeit einer Anpassung anhand einer vorhandenen Störung, deren Frequenz unter $1 \mathrm{~Hz}$ liegt, gezeigt werden. Die wahrscheinlichste Quelle dieser Störung ist der von uns verwendete Laser (Koheras Basik E15), da er seine Wellenlänge über eine interne Temperatur regelt. Da diese durch äußere Einflüsse sowie die Wärmeentwicklung des Lasers im Bereich von $0,1 \mathrm{~K} / \mathrm{min}$ schwankt, schwankt auch die Wellenlänge im Bereich einiger Pikometer. Zur Visualisierung sind in Abb. 5 drei Messungen dargestellt. Diese zeigen jeweils die Auslenkung im demodulierten Zeitsignal. Darin ist die Auswirkung der temperaturabhängigen Wellenlängenänderung gut erkennbar. In Grün ist der ideale Fall dargestellt, bei dem die

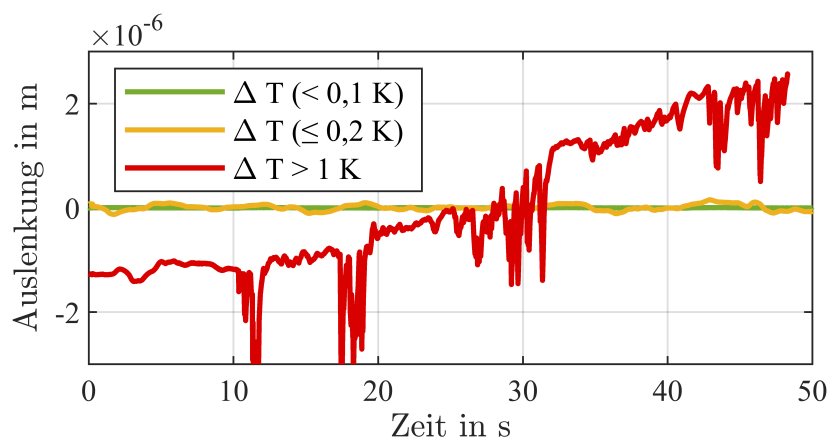

Abb. 5: Demoduliertes Zeitsignal zur Darstellung des Einflusses der temperaturabhängigen Wellenlängenänderung auf die detektierte Auslenkung. 
Schwankung der Temperatur so gering ist, dass die Wellenlänge nahezu konstant gehalten wird. In Gelb ist der reale Fall nach einigen Minuten Betriebszeit dargestellt. Über eine längere Messung kann sich die Temperatur um $0,1 \mathrm{~K}$ bis $0,2 \mathrm{~K}$ ändern und es resultiert eine größere Auslenkung. In Rot ist ein unrealistischer Extremfall zur Anschauung dargestellt. Hierbei wurde die Temperatur durch externe Wärmezufuhr um $1 \mathrm{~K}$ angehoben. Dabei ändert sich die Wellenlänge um mehr als 100 pm und erzeugt so eine starke Auslenkung im demodulierten Zeitsignal.

Durch die Notwendigkeit eines mobilen Aufbaus ist der Laser im DLDV integriert und deshalb kann eine Temperaturregelung nur teilweise umgesetzt werden. Da die resultierende Störung durch die Wellenlängenänderung im für uns relevanten Frequenzbereich liegt, muss der Einfluss ohne eine Temperaturreglung so weit wie möglich reduziert werden. Methoden dazu werden in den folgenden Abschnitten beschrieben.

\subsubsection{Laufzeiten optischer Signale}

Bei einem LDV verlässt der Referenzstrahl das Gerät nicht und besitzt deshalb eine klar definierte Weglänge. Bei einem DLDV werden hingegen sowohl Mess- als auch Referenzstrahl nach außen geführt, wodurch es möglich ist, die Differenz dieser Wege zu minimieren. Der Einfluss der Weglängenanpassung und in welchem Maße diese vorgenommen werden muss, wird im folgenden Abschnitt erläutert.

Ein Weglängenunterschied beider Strahlen von $1 \mathrm{~m}$ führt mit der Lichtgeschwindigkeit $c=2,998 \cdot 10^{8} \frac{\mathrm{m}}{\mathrm{s}} \mathrm{zu}$ einem Laufzeitunterschied von ungefähr 3,34 ns. Wenn zwischen beiden Strahlen ein Laufzeitunterschied besteht, kann dieser mit $t_{2}=t+\Delta t$ angenommen werden. Am Detektor überlagern sich die elektrischen Felder $E_{M}$ des Mess- und $E_{R}$ des Referenzstrahls. Im Fall eines DLDV ergeben sich die Felder zu

$$
\begin{aligned}
E_{M}(t) & =E_{m} \cos \left(2 \pi f_{1} t-\varphi_{M}(t)\right) \\
E_{R}(t) & =E_{r} \cos \left(2 \pi f_{2} t_{2}-\varphi_{R}\left(t_{2}\right)-\varphi_{0}(\Delta t)\right)
\end{aligned}
$$

mit $f_{1}=f_{0}+f_{b}, f_{2}=f_{0}, f_{0} \approx 193 \mathrm{THz}$ (Lichtfrequenz bei $1550 \mathrm{~nm}$ ), $f_{b}=40 \mathrm{MHz}$ (Frequenzänderung durch Bragg-Zelle; entspricht der Trägerfrequenz), $\varphi_{R}\left(t_{2}\right), \varphi_{M}(t)$ (Phasenverschiebung des Referenz- bzw. Messstrahls durch den Doppler-Effekt), $\varphi_{0}(\Delta t)$ (Phasenverschiebung durch Laufzeitunterschied) und $E_{m}, E_{r}$ (Amplituden der Felder). Damit lässt sich das Detektorsignal der Photodioden aus dem Gesamtfeld bestimmen zu

$$
i_{p} \propto \frac{E_{m}^{2}}{2}+\frac{E_{r}^{2}}{2}+E_{m} E_{r} \cos (\Phi(t))
$$

[8] mit $i_{p}$ (Photodiodenstrom). Relevant zum Verständnis ist dabei das Argument $\Phi(t)$ im Kosinus. Mit $f_{\Delta}=$ $f_{0}(t+\Delta t)-f_{0}(t), \varphi_{\Delta}=\varphi_{R}(t+\Delta t)-\varphi_{M}(t)$ ergibt sich dafür

$\Phi(t)=2 \pi f_{b} t+2 \pi f_{\Delta} \Delta t+\varphi_{0}(\Delta t)+\varphi_{\Delta}-\varphi_{M}(t)+\varphi_{R}(t)$.

Für kleine Abstände zwischen Mess- und Referenzsstrahl, läuft $\varphi_{R}(t)-\varphi_{M}(t)$ gegen Null. Bei $\Delta t=0$ werden alle anderen Terme, bis auf den mit der Trägerfrequenz $f_{b}$, ebenfalls zu Null. Sobald ein Laufzeitunterschied existiert, bewirkt dieser auch eine Frequenz- und Phasenänderung des Detektorsignals. Damit eine Messung möglichst störungsfrei ist, muss demnach der Laufzeitunterschied möglichst gering sein. Dies kann nur durch die Anpassung der Weglängen erreicht werden. Um den Einfluss einer solchen Anpassung zu demonstrieren, werden jeweils einige Messungen mit Positionierung der Strahlen auf Spiegeln und Variation der Weglängen durch Verschiebung dieser vorgenommen. Um den Effekt zu verdeutlichen, wurde die Wellenlänge des Lasers mit einer Frequenz von $400 \mathrm{~Hz}$ moduliert. Die Ergebnisse dieser Messungen sind in Abb. 6 dargestellt. Bei einem Laufzeitunterschied wird die Modu-

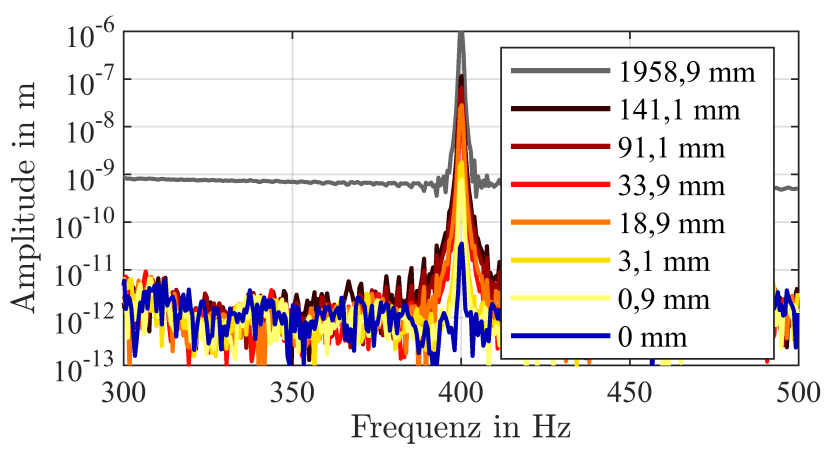

Abb. 6: Vergleich der Amplitude mit modulierter Wellenlänge bei $400 \mathrm{~Hz}$ mit unterschiedlichen Weglängen, angegeben ist jeweils die Differenz der Weglängen beider Strahlen.

lation, die in diesem Fall als $f_{\Delta}$ in Gl. 2 verstanden werden kann, als Frequenzänderung im Detektorsignal erfasst und verursacht eine große Amplitude im Frequenzspektrum.

\subsubsection{Laufzeiten elektrischer Signale}

Ein weiterer Störfaktor ist zudem die Schwankung der Trägerfrequenz $f_{b}=40 \mathrm{MHz}$, die in einem Signalgenerator erzeugt und über einen Verstärker zur Bragg-Zelle geführt wird. Zur Demodulation wird das Signal aus dem Signalgenerator (Referenzsignal) sowie das verstärkte Signal des 
Detektors (Messsignal) zu einem Analog-Digital-Umsetzer (ADU) geführt und digitalisiert. Bei der Übertragung der elektrischen Signale sind Übertragungsgeschwindigkeiten, wie z. B. in [9] beschrieben, zu beachten. Durch unterschiedliche Übertragungswege und Komponenten sowie die zusätzliche Verzögerung des Messsignals durch die optische Weglänge, besteht ein Laufzeitunterschied des Messund des Referenzsignals. Die Differenz beträgt dabei bei unserem Aufbau mit 16 ns etwas weniger als die Dauer einer Periode der Trägerschwingung von $25 \mathrm{~ns}$.

Die Asynchronität wurde in unserem Aufbau kompensiert, indem das Referenzsignal verzögert wird. Durch die Abtastrate des ADU von $125 \mathrm{MHz}$ beträgt die SampleLänge $8 \mathrm{~ns}$. Das beste Ergebnis wurde dabei mit einer Verzögerung von 3 Samples, die $24 \mathrm{~ns}$ entsprechen, erreicht und ist in Abb. 7 dargestellt. Die Abweichung zur erwarteten Differenz von 16 ns lässt sich durch weitere Verzögerungen, einen abweichenden Verkürzungsfaktor sowie die grobe Schrittweite erklären. Durch die angepasste Verzögerung sind der grundsätzliche Rauschpegel und andere Störungen nur gering betroffen. Es wird allerdings eine Verringerung des Einflusses der Netzfrequenz von $50 \mathrm{~Hz}$ im Frequenzspektrum erreicht. Die eigentliche Schwankung des generierten $40 \mathrm{MHz}$-Signals wird demnach nicht erfasst oder hat nur einen kleinen Einfluss auf den Rauschpegel.

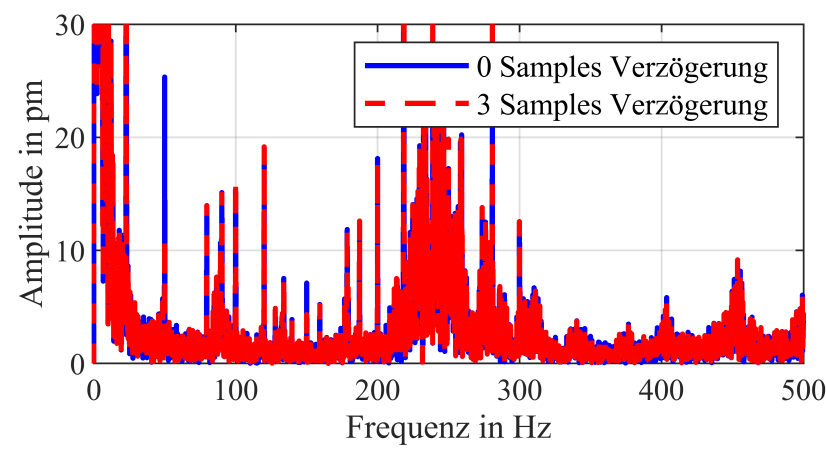

Abb. 7: Verringerung des Einflusses der Netzfrequenz $(50 \mathrm{~Hz})$ auf den Rauschpegel durch Synchronisation der Signale aus dem Signalgenerator und vom Detektor.

\section{Zusammenfassung}

Es wurde anhand einer LDV-Messung gezeigt, dass bei hohen Temperaturen der Rauschpegel durch den Einfluss von Hitzeflimmern stark ansteigt. Mithilfe eines einfachen Aufbaus wurde bewiesen, dass dieser Einfluss durch das differentielle Messen mit einem selbst entwickelten DLDV verringert werden kann. In diesem Zusammenhang wurde auf Limitationen bzw. einzuhaltende Bedingungen, insbesondere bezogen auf den Abstand des Mess- und des Referenzsstrahls, eingegangen. Zusätzlich wurde das Problem der temperaturabhängigen Wellenlängenänderung des Lasers erläutert, wobei die Relevanz der Weglängenanpassung aufgezeigt und anhand einer Messreihe bestätigt wurde. Für differentielles Messen ist es außerdem wichtig, ein Modell für die Entstehung und die Größe der Zellen, in denen Brechungsindexschwankungen auftreten, zu erstellen, oder experimentell festzustellen.

Zur weiteren Minimierung des Rauschpegels müssen zusätzliche Störfaktoren wie noch bestehende Weglängendifferenzen der Laserstrahlen sowie elektrische Einflüsse und Vibrationen durch Umwelteinflüsse identifiziert und analysiert werden.

Danksagung: Die vorgestellten Arbeiten wurden von der Deutschen Forschungsgemeinschaft (DFG) im Rahmen des Projekts Chemische Expansion von Praseodym-CerMischoxidschichten bei hohen Temperaturen finanziert (Förderkennzeichen FR1301/31-1 und RE3980/3-1).

\section{Literatur}

[1] L. C. Andrews und R. L. Phillips. Laser Beam Propagation through Random Media. SPIE, Sept. 2005.

[2] A. Dräbenstedt, J. Sauer und C. Rembe. Remote-sensing vibrometry at $1550 \mathrm{~nm}$ wavelength. AIP, 2012.

[3] A. Dräbenstedt, X. Cao, U. Polom, F. Pätzold, T. Zeller, P. Hecker, V. Seyfried und C. Rembe. Mobile seismic exploration. AIP Conference Proceedings, 1740(1):030001, 2016.

[4] J. C. Owens. Optical refractive index of air: Dependence on pressure, temperature and composition. Applied Optics, 6(1): 51, Jan. 1967.

[5] C. Rembe und A. Dräbenstedt. Laser-scanning confocal vibrometer microscope: Theory and experiments. Review of Scientific Instruments, 77(8):083702, Aug. 2006.

[6] S. Schmidtchen, H. Fritze, S. Bishop, D. Chen und H. L. Tuller. Chemical expansion of praseodymium-cerium oxide films at high temperatures by laser doppler vibrometry. Solid State lonics, 319:61-67, June 2018.

[7] F. V. Shugaev, L. S. Shtemenko, O. A. Nikolaeva, T. I. Arsenyan, N. A. Suhareva und A. P. Sukhorukov. Modeling of laser beam propagation through turbulence. SPIE, Oct. 2014.

[8] H. Steger, M. Wörtge, G. Siegmund und C. Rembe. Measuring MEMS in motion by laser doppler vibrometry. In Optical Science and Engineering, S. 245-292. CRC Press, July 2006.

[9] F. Strauß. Leitungstheorie. In Grundkurs Hochfrequenztechnik, S. 57-116. Springer Fachmedien Wiesbaden, 2016. 